\title{
Gastric Glomus Tumour Presenting with Massive Upper GI Bleeding: A Challenging to Diagnose and Treat Tumour
}

\author{
Ahmad Ayash ${ }^{1}$, Nasrein Elkomy $^{1}$, Muneera Almohannadi ${ }^{1}$, and Saad Alkaabi ${ }^{2}$ \\ ${ }^{1}$ Hamad Medical Corporation \\ ${ }^{2}$ Hamad Medical Corp
}

February 4, 2022

\begin{abstract}
Glomus tumours are infrequent benign tumours originate from the glomus body and can be found anywhere in the body. We report the case of a 61 year-old patient who presented with massive upper GI bleeding secondary to an ulcerated gastric glomus tumour that eventually treated with surgical wedge resection.
\end{abstract}

\section{Introduction}

Glomus tumours (GTs) are rare benign tumours arising from modified smooth muscle cells of the glomus body [1]. GTs usually occur in the distal extremities, particularly in the nail beds of the fingers. However, they can occur anywhere in the body, including in the gastrointestinal (GI) tract, and most GI tumours are located in the gastric antrum [1]. Gastric glomus tumours (GGTs) are a rare cause of upper GI bleeding. GGTs usually present with a wide variety of symptoms, such as haematemesis, melena, and dyspepsia, but sometimes they are asymptomatic and discovered incidentally [2].

We report a case of GGT that presented with life-threatening upper GI bleeding, which was initially treated endoscopically and then ultimately surgically resected as a definitive treatment.

\section{Case presentation}

The case was a 61-year-old patient. The significant aspects of his medical history were as follows: type 2 diabetes mellitus, hypertension, end-stage renal disease on regular haemodialysis, coronary artery disease, and rectal cancer status post-abdominoperineal resection with permanent colostomy.

He was referred to the endoscopy unit by his oncologist for workup of iron deficiency anaemia, with a history of passing dark stools intermittently for the past few weeks.

On clinical examination, the patient was haemodynamically stable. There was pallor with a normal abdominal examination. There was no evidence of melena at the time of examination. Laboratory tests revealed: haemoglobin $=6.4 \mathrm{~g} / \mathrm{dL}$, platelet count $=357,000 / \mu \mathrm{L}, \mathrm{INR}=1$, urea $=23.5 \mathrm{mmol} / \mathrm{L}$ and creatinine $=342$ $\mu \mathrm{mol} / \mathrm{L}$. Liver function test results were normal.

Esophagogastroduodenoscopy (EGD) revealed an approximately $3 \mathrm{~cm}$ subepithelial lesion in the antrum with a clean-based ulcer in the centre. There was no evidence of altered or fresh blood in the stomach. A colonoscopy was performed through the colostomy and was normal. See figure 1.

Subsequently, an endoscopic ultrasound (EUS) was conducted and revealed a hyperechoic antral lesion originating from the second, third layer. The EUS findings were suggestive of lipoma. See figure 2. 
An abdominal CT scan with contrast was performed. The images showed a well-defined subepithelial soft tissue mass lesion in the antrum measuring $3 \mathrm{~cm}$ and bulging in the stomach lumen. The lesion showed heterogeneous post-contrast enhancement. There was no definite invasion of the adjacent structures. See figure 3. A multidisciplinary team meeting was held between the upper GI surgeon and the gastroenterologist. They agreed to observe the patient and treat him conservatively.

Six weeks later, the patient presented to the emergency department with a one-day history of dizziness and haematemesis. The patient deteriorated rapidly in the emergency department within a few hours. On clinical examination, he was drowsy and haemodynamically unstable, with signs of shock. His blood pressure was $84 / 52 \mathrm{mmHg}$, heart rate was $100 \mathrm{BPM}$, respiratory rate was 29 breaths/min, and O2 saturation was $90 \%$ on room air. The colostomy bag contained melena.

Laboratory tests revealed a haemoglobin level of $3.2 \mathrm{~g} / \mathrm{dL}$, a platelet count of $155,000 / \mu \mathrm{L}$, an INR of 1.2 , urea at $46 \mathrm{mmol} / \mathrm{L}$ and creatinine at $511 \mu \mathrm{mol} / \mathrm{L}$. He was intubated and admitted to the medical intensive care unit as a case of upper GI bleeding with haemorrhagic shock. He received a packed red blood cell transfusion and was started on an intravenous pantoprazole infusion. An urgent bedside EGD was performed and revealed that the previously observed antral subepithelial lesion had blood oozing from the ulcer at the tip of the lesion. Two endoclips were deployed and haemostasis was achieved.

To prevent further life-threatening GI bleeding, the decision was made to surgically remove the lesion.

After receiving an explanation of the benefits and risks of the surgery, the patient agreed to proceed with the procedure. Subsequently, he underwent an open wedge resection of the stomach. Histopathologic examination confirmed the diagnosis of GGT, with free excision margins. There was a low mitosis rate, with no atypia. Ki67 showed a proliferation index of approximately $10 \%$. Immunohistochemical staining was strongly positive for smooth muscle actin, calponin, caldesmon, and synaptophysin, and was negative for CD 117, CD 34, CD 56, S 100, CK 20 and chromogranin. The patient was doing well after nine months of follow-up post-surgery. There was no evidence of GI bleeding again and his haemoglobin returned to baseline levels.

\section{Discussion}

GTs are rare mesenchymal tumours arising from the neuromyoarterial glomus. They account for $1 \%$ of gastrointestinal stromal tumours (GISTs) [3]. Despite being reported in the literature in early 1951 by Kay et al., few cases have been reported since, and there are no established guidelines for their management [4]. Most GGTs are solitary and located in the gastric antrum; they are rarely reported in multiples [5]. Moreover, they might present with abdominal pain, gastrointestinal bleeding, or perforation [6]. It can also be found incidentally, with no symptoms [7]. Although GGTs have distinctive macroscopic and microscopic morphology, they have some overlapping features with other GISTs and carcinoid tumours [8]. This case demonstrates that the diagnosis of GGT poses a challenge, given that GGTs lack typical endoscopic and clinical features and share many features with other gastric subepithelial tumours. However, upper GI bleeding is known to be associated with GGTs; it has not been reported to be life threatening or in nonulcerative GGTs.

In CT scans, GGTs appear as well circumscribed subepithelial masses that have strong arterial phase enhancement, a progressive filled-in enhanced pattern, and prolonged enhancement during multiphasic scans. Unfortunately, CT imaging cannot differentiate between GGTs and other mesenchymal tumours [9]. For definite diagnosis, EUS-FNA with immunohistochemical examination must be performed. In EUS, GGTs appear as hypoechoic, well-circumscribed lesions in the submucosa and/or muscularis propria layers [10]. Pathological examination of GGTs shows small uniform epithelioid cells that are positive for smooth muscle actin and calponin and exhibit PAS-positive basement membranes. The cells are negative for desmin, cytokeratin (AE1/AE3b), S-100, creatine kinase, C-KIT (CD-117), CD34, DOG1 (K9), chromogranin A, p53 and neuron-specific enolase [11,12].

The treatment of choice for GGT is surgery with negative margins, which may include resection of the 
tumour, subtotal gastrectomy, or wedge resection [13].

\section{Conclusion}

In this case report, GGT was initially misdiagnosed as a lipoma and later presented with a life-threatening GI bleed that was ultimately treated with surgical resection of the tumour. GGT is a rare disease with limited published knowledge, and there are no definitive guidelines for diagnosis and treatment. To date, the course of GGT management has been left to the physician's discretion.

\section{Ethical Approval}

Case report was approved (MRC-04-21-641) by the IRB and Medical Research Centre of Hamad Medical Corporation, Doha, Qatar.

\section{Consent}

Informed consent was taken from the patient.

\section{Conflict of Interest}

The authors declare that they have no conflicts of interest.

\section{Authors' Contributions}

Ahmad Ayash and Nasrein Elkomy reviewed the literature, drafted and edited the manuscript, and approved the final manuscript. Muneera Jassim Al-Mohannadi, and Saad Rashid Al-Kaabi reviewed the literature and edited and approved the final manuscript. The manuscript was prepared according to ICJME guidelines and CARE guidelines for reporting case reports.

\section{References}

1-Tsuneyoshi M, Enjoji M. Glomus tumor: A clinicopathologic and electron microscopic study. Cancer. 1982, 50:1601-1607. (reference for definition)

2-Chen KB, Chen L. Glomus tumor in the stomach: A case report and review of the literature. Oncol Lett. 2014, 7(6):1790-1792. doi:10.3892/ol.2014.1986

3-Miettinen M, Paal E, Lasota J, Sobin LH. Gastrointestinal glomus tumors: A clinicopathologic, immunohistochemical, and molecular genetic study of 32 cases. Am J Surg Pathol. 2002, 26:301-311.

4-Kay S, Callahan WP, Murray MR, Randall HT, Stout AP. Glomus tumors of the stomach. Cancer. 4(4):726-736.

5-Kanwar YS, Manaligod JR. Glomus tumor of the stomach. An ultrastructural study. Arch Pathol. 1975, 99:392-397.

6-Haque S, Modlin IM, West AB. Multiple glomus tumors of the stomach with intravascular spread. Am J Surg Pathol. 1992, 16:291-299.

7-Wu M, Zhou T, Cao D, Qu L, Cao X. Glomus tumor of the stomach. A case report. Medicine. 2018, 97(45):e13132.

8-Fang HQ, Yang J, Zhang FF, Cui Y, Han AJ. Clinicopathological features of gastric glomus tumor. World J Gastroenterol. 2010, 16:4616-4620.

9-Hu SD, Hu DM, Huang W, Chen KM, Song Q. CT and clinical characteristics of GGT. Journal of Digestive Diseases. 2014, 15:477-482. https://doi.org/10.1111/1751-2980.12172.

10-Miettinen M, Paal E, Lasota J, Sobin LH. Gastrointestinal glomus tumors: A clinicopathologic, immunohistochemical, and molecular genetic study of 32 cases. Am J Surg Pathol. 2002, 26:301-311. 
11-Lee HW, Lee JJ, Yang DH, Lee BH. A clinicopathologic study of glomus tumor of the stomach. J Clin Gastroenterol. 2006, 40:717-720.

12-Folpe AL, Fanburg-Smith JC, Miettinen M, Weiss SW. Atypical and malignant glomus tumors: Analysis of 52 cases, with a proposal for the reclassification of glomus tumors. Am J Surg Pathol. 2001, 25:1-12.

13-Wang X, Hanif S, Wang B, Chai C. Management of gastric glomus tumor: A case report. Medicine. 2019, 98:e16980. 10.1097/MD.0000000000016980.

Figure 1. showing endoscopic image of the gastric glomus tumour

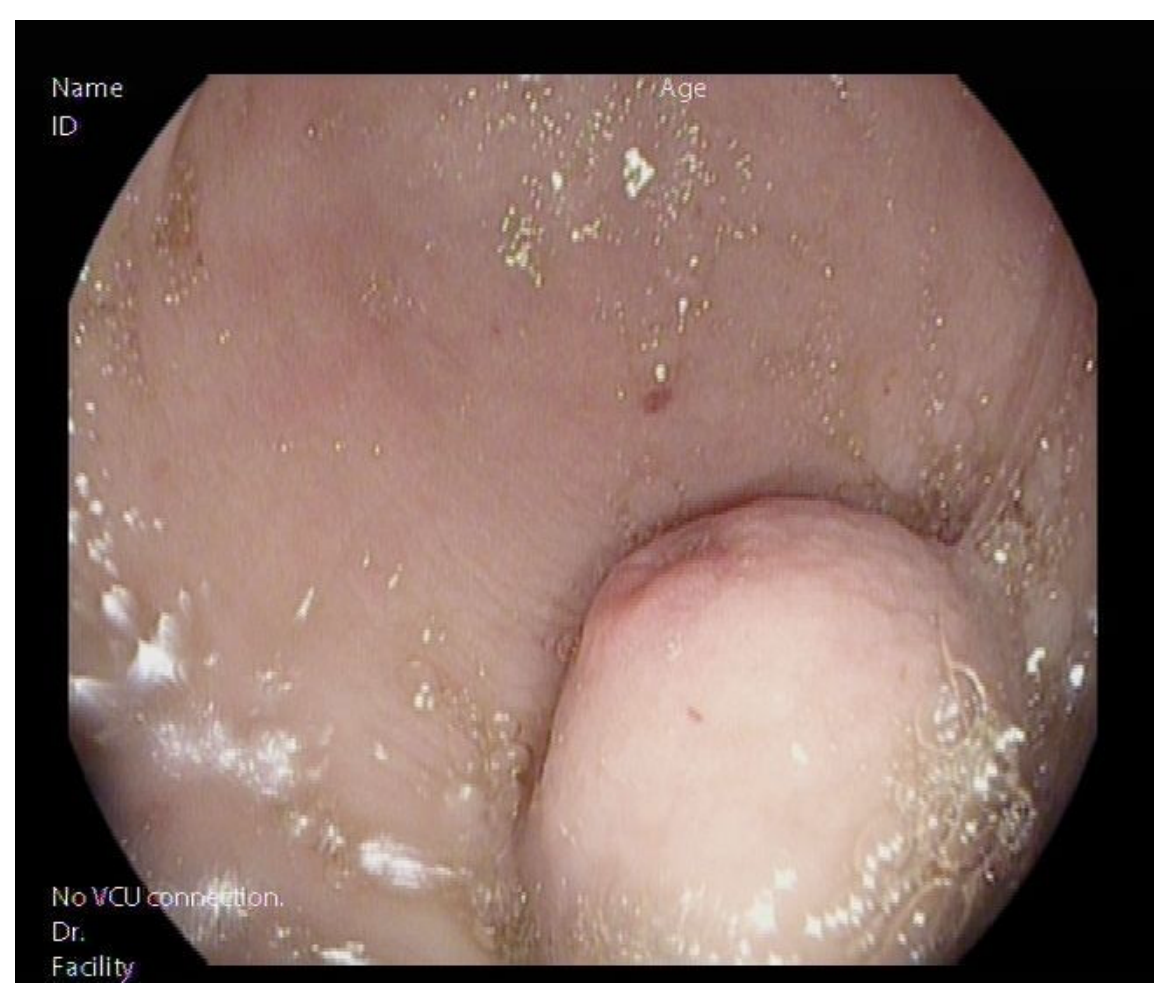

Figure 2. showing the EUS image of the tumour arising from the $2^{\text {nd }}, 3^{\text {rd }}$ layer 


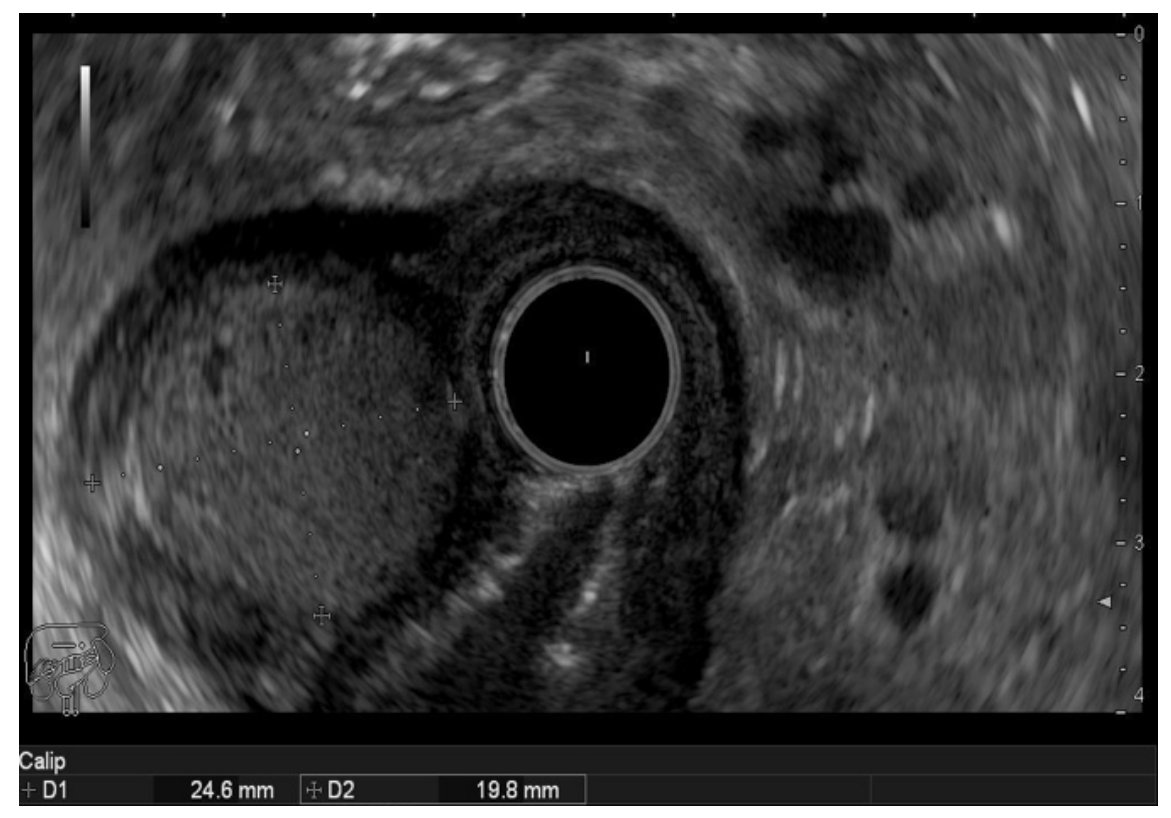

Figure 3. Abdomen CT scan with contrast (Axial image): shows the subepithelial tumour in the gastric antrum.

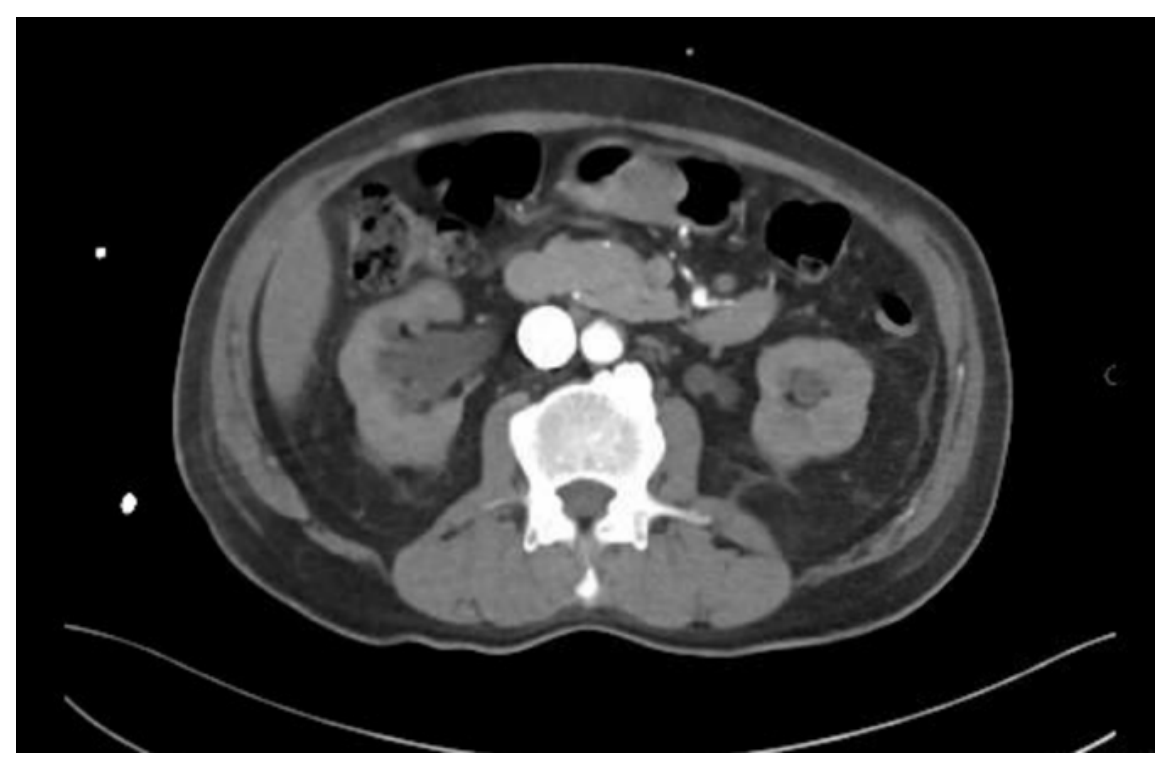

